\title{
Navigating assisted reproduction treatment in the time of COVID-19: concerns and considerations
}

\author{
Mara Simopoulou ${ }^{1,2}$ (1) $\cdot$ Konstantinos Sfakianoudis ${ }^{3} \cdot$ Polina Giannelou $^{1,3} \cdot$ Anna Rapani $^{1,2}$. \\ Charalampos Siristatidis ${ }^{2} \cdot$ Panagiotis Bakas $^{2} \cdot$ Nikolaos Vlahos $^{2} \cdot$ Konstantinos Pantos $^{3}$
}

Received: 3 August 2020 / Accepted: 7 September 2020 / Published online: 16 September 2020

(C) Springer Science+Business Media, LLC, part of Springer Nature 2020

\begin{abstract}
The COVID-19 pandemic has fueled numerous debates in the field of assisted reproductive technology (ART) as the effect of SARS-CoV-2 on pregnancy and infancy is still considered uncharted territory. Various theses and recommendations on what optimal practice is have emerged, as evidenced by surveys, webinars, and recent publications. ART specialists are faced with dilemmas in light of the lack of concrete scientific evidence required to pave the way towards future safe practice. Meanwhile, infertile couples were similarly left in limbo unable to exercise their reproductive autonomy unlike fertile couples-where achieving a pregnancy via natural conception is a matter of decision. ART treatment being classified as non-essential has only recently re-started, facing new challenges while enabling pregnancy at a time of uncertainty. This article highlights matters of bioethical nature to be considered in the ART world at the time of COVID-19 while presenting an all-inclusive critique of the current status. When pursuing pregnancy through IVF treatment during the pandemic, distancing and caution have the lead role in an effort to defend the health of the intended parents and future children. To promote patient autonomy along with our ethical, moral, and legal duty towards our patients, emphasis should be given on ascertaining shared decision-making, and ensuring that an appropriate all-inclusive informed consent is signed prior to initiating any IVF treatment.
\end{abstract}

Keywords Reproductive technologies $\cdot$ Public health $\cdot$ Right to health care $\cdot$ Professional ethics

\section{ART as "non-essential" care, the response of the ART field, and discrepancies in management}

As the COVID-19 pandemic introduced an unprecedented strain on the global health care system, numerous countries issued directives and instructions suggesting that nonessential care must be discontinued, in an effort to reorganize

Mara Simopoulou

marasimopoulou@hotmail.com

1 Department of Physiology, Medical School, National and Kapodistrian University of Athens, Mikras Asias, 11527 Athens, Greece

2 Assisted Conception Unit, 2nd Department of Obstetrics and Gynecology, Aretaieion Hospital, Medical School, National and Kapodistrian University of Athens, Vasilissis Sofias,

11528 Athens, Greece

3 Centre for Human Reproduction, Genesis Athens Clinic, Papanikoli, 15232 Athens, Greece resources to fight the spreading of SARS-CoV-2 [1]. The definition "non-essential care" triggered a rise of concern on behalf of special interest groups. Considering that the field of assisted reproductive technology (ART) does not constitute a first-line field of Medicine, in comparison with the field of oncology, where typically cases require urgent management, various opinions have been raised with respect to discontinuing or not proceeding at all to fertility treatments. This generated a division of views with regard to what is deemed necessary and essential in healthcare discerning between elective and non-elective and how straightforward and bioethically "correct" it is to draw this theoretical line. Projection of the impact on the overall fragile and susceptible health care system certainly served as a driver in forming respective views.

Following an initial estimate of the risk, reflexes were activated urging the field to respond. The series of events that followed and the attitudes adopted by the field of ART with respect to providing services were formed in response firstly to the fact that we were "navigating unchartered waters" and secondly in response to the guidelines issued [2]. The reaction 
of the ART field to COVID-19 being perceived as a harmful event interestingly resembled the "fight," "flight," or "freeze" response. The "flight" attitude referred to treatment cessation all together, followed by the somewhat literal response of "freeze" described as the decision to avoid embryo transfers during the initial stages of the pandemic and proceed with cryopreserving any generated embryos in the laboratory. This led to the "fight" response, confirmed by the restart of providing in vitro fertilization (IVF) services employing COVID-19-fit protocols.

During these unsettling times, fertility treatments were initially suspended across certain countries due to the COVID-19 pandemic in accordance with the recommendation of the British Fertility Society, American Society for Reproductive Medicine (ASRM), and European Society of Human Reproduction and Embryology (ESHRE). With fertility patients left in limbo and the Assisted Conception Units not treating new patients, uncertainty and anxiety have been expressed [3].

What the field has experienced over the last few months since the emergence of the pandemic are discrepancies in the manner and the extent that ART treatment is offered. Both public and private centers have been equally affected since no difference between the two in terms of the status of providing services has been documented, with the exception of an earlier restart of activities in private centers of some countries [4]. Regarding both the public and private sectors, fertility services are offered following instructions and recommendations that may be open to interpretation, inevitably leading to discrepancies in practice. Newly published data on medically assisted reproduction activities in Europe during the COVID19 pandemic noted these discrepancies and emphasized on the lack of a common approach in the clinical field of ART [2]. This is still an issue that remains to be addressed with the pandemic still raging, at a time when the value of consistency cannot be stressed enough.

\section{Recommended guidelines for restarting practice and emerging concerns}

Highly esteemed societies issued recommendations for practice and announced the restart of ART treatment. Prognostic stratification coupled with apt identification of patients requiring timely treatment was highlighted as the means to ensure an optimal and gradual restart of ART services [5]. Following "restrictions' relaxation," and in an effort to convey safety in practice, certain strategies have been adopted. These indicatively include patients' and personnel's recurrent testing, promotion of tele-healthcare, personal protective equipment (PPE) use, along with introducing employment of the wellknown triage questionnaire addressing both the personnel and the patient perspective, while employing strict safety protocols for both patients and personnel. The question arising is "will this be enough?" With IVF treatment by definition being a product of "team effort" between the medical, the nursing, and the embryology teams, how can the concept of "individualized care" be realized is a challenge from any perspective. Definitive guidance on embryos' and gametes' management is yet to be fully elucidated, and the risks entailed in case of contamination have yet to be explored. The hypothesis that the ART field can ensure safe and effective practice by implementing the valued proposals submitted hitherto still needs to be tested. The field is called to address a triad of issues with respect to patient safety, personnel safety, and finally gamete and embryo safety in the andrology and embryology laboratory and the cryopreservation bank. To add to the intricacy of the equation, consider the intertwined relationships between these three levels, and the amplified complexity of the matter is instantly highlighted. As the COVID-19 pandemic still progresses more so in some parts of the world, infertility treatment perseveres raising bioethical concerns and skepticism.

In the newly found COVID-19 era of "zoom fatigue" and non-stop webinars, it appears that ART professionals are contemplating whether adopting optimal protocols in the IVF laboratory could provide a safety net. This is indicated as numerous surveys have been promoted and undertaken and special interest webinars targeting clinical embryologists and physicians in ART are scheduled around the clock to provide us with insight and feedback on attitudes and understanding of risk. Employment rights have been compromised during this time and mitigation processes may be subject to interpretation of the recommendations and guidelines leading to uncertainty and stress experienced by the professionals in ART along with other fields of Medicine. This describes a highly complex and demanding situation one where, in the interest of communal health, employees were expected to sustain a considerable and unexpected financial strain extending to their ability to support themselves and their families [6]. The reinstatement of fertility clinics was certainly not the outcome of the influx of comforting robust data and knowledge on COVID-19; hence, it is inevitable to raise concerns regarding the timing along with the safety of such a decision in its entirety. Nonetheless, with the world entering a phase of restart on all socioeconomic levels, ART treatment following appears to be the way forward.

\section{The case for patient prioritization}

ART is a special field in medicine. Women undergoing fertility treatment may not always be infertile and infertility may not always be related to a medical condition. Should we be advising our patients to wait - should they have the luxury of time - to address infertility at a more peaceful time? In the 
prospect of the face of the pandemic becoming a familiar one, how should we consult our patients taking into consideration that the demand for fertility restoration is present and consistent? Oncological patients along with patients of advanced maternal age and compromised ovarian reserve should be prioritized as poor prognosis patients as supported by published data [7]. Prioritization should also be recommended for all time-sensitive patients experiencing anxiety with regard to planning their treatment schedule [8]. Further to that, it has been reported that men similarly may be detrimentally affected in the prospect of postponing diagnostic semen analysis and respective cryopreservation to the bank. With ART services becoming unavailable, biological parenthood for men may be equally compromised [9]. In the words of Professor Berger providing a valuable thesis on the general situation, "control measures should be equitable and inclusive" [6]. On the other hand, and on the matter of timing being of the essence for diminished ovarian reserve (DOR) patients, interesting data has just been published by Romanski and colleagues providing a retrospective data analysis on DOR patients investigating the effect of receiving treatment with a 6-month delay. The study concluded that a short-term delay in treatment that may be stemming from reasons of medical, logistic, or financial nature does not exert any negative effect on treatment outcomes [10]. With contradicting theses on how a potential delay may affect outcome, and whether a 6-month period is considered a short-term delay, we may be back to the acknowledgment that the truth lies somewhere in the middle. It may be so that our attention should be focused on documenting patients' views on postponing treatment. It is imperative for this perspective to be accounted for. This will provide us with valuable insight from another point of view that can equally help shape future practice. Furthermore, such data can guide us on matters of educating and raising awareness regarding the association of infertility to age and aging. Our duty to future generations may perhaps be to prevent our "future patients" from becoming our "future patients"-while there is still time. At a time of crisis, it is anticipated that the human race contemplates on matters of importance. Fecundity and fertility and the planet's population certainly make for topics worth analyzing.

\section{The matter of patients' psychological state during the pandemic}

Following the initial suspension of IVF treatments, patients understandably experienced insecurity and a state of heightened stress. The psychological state of infertile patients during these times is certainly a topic that merits further investigation as data accumulates. Perhaps the compromised psychological state of IVF patients during the pandemic and the need for this to be addressed by the IVF field should be accordingly underlined, and - albeit it may not be viewed as a priority at a time of crisis - it should not be underrepresented when recommendations are issued. A plethora of newly published scientific articles aiming to shed light on this pandemic have come to the rescue. A systematic review [11] of the current evidence showcased a clear impact of the COVID-19 pandemic on the mental health of the general population, health workers, and patients who seek various treatments during this period [12-14]. Considering the given and acknowledged psychological strain and the burdened mental well-being of couples pursuing infertility treatment that has been showcased [15-17], it becomes evident that infertile couples subjected to IVF treatment during the pandemic may face a heightened psychological distress under the circumstances of this global health crisis [5]. With infertile patients being more susceptible to suffer from anxiety than in other medical disciplines where time may not be of the essence, psychological support by clinicians and embryologists is recommended [8]. In a recent survey conducted by ASRM, including a total of 518 patients, the authors concluded that considering the severity of this global situation the physical, financial, and emotional impact is significant and should not be underestimated for these patients [18]. As data is limited, in order to evaluate the true long-term effect on IVF patients' mental state, we need to allow time for further data to accumulate in order to extract safe conclusions and not extrapolations. Meanwhile, this issue remains open and in progress adding another level of complexity to designing optimal ART practice during COVID-19.

\section{Implications of exercising the right to reproduction employing ART during the pandemic}

The impact of COVID-19 on embryos and the potential underlying mechanisms affecting progress of pregnancies remain to be further investigated Hitherto, research concerning the impact of COVID-19 on pregnancy and embryos is still fluid, despite the influx of new data emerging. On COVID-19 and pregnancy, the Centers for Disease Control and Prevention (CDC) has not hitherto reported any issues regarding the first and second trimesters; nonetheless, conclusive data still eludes us [19]. Some evidence suggests that COVID-19 may present notable perils for pregnant women [20, 21]; thus IVF specialists should be extremely cautious during the decision-making process. Further to that, the prospect of medication in case of an infection during the first and second trimesters of gestation involving certain drugs and protocols is certainly contraindicated [7]. Apart from the lack of robust foolproof data on the risks entailed in IVF treatment, the fact that COVID-19's impact during pregnancy and infancy still eludes us to a large extent raises valid bioethical concerns with regard to the fact that IVF treatment promotes pregnancy at an uncertain time. Albeit a bold statement, this may be a harsh reality 
so the cliché of further studies being required to delineate the identity of this pandemic is inevitable and essential. Where does the ART field stand in the midst of all of that is? And how could that affect infertile patients towards exercising their right to procreate, especially when natural conception is certainly not subject to any medical recommendation with regards to being avoided? Should ART indications change during a pandemic especially as there is no concomitant recommendation for women to avoid pregnancy through sexual intercourse? Should pregnancy be promoted for the reproductively challenged couples and if not why is it that no Scientific society has issued a respective concern for pregnancies ensued via natural conception? Following in that mindset, why should IVF patients be prevented from pursuing a pregnancy? From an ethical point of view, it has already been voiced that declining the infertile patients' right to have access to IVF treatment in order to pursue a pregnancy — even at the time of a pandemic - would be an act of discrimination against fertile couples where natural conception is a matter of decision [7]. COVID-19's impact on opting for and receiving IVF treatment showcases that this lockdown can be symbolically paralleled to a lockdown of options. Nonetheless, it remains to be seen whether, and to what extent, the pandemic affected the decision of couples to conceive naturally or via IVF treatment. Along these lines, in order to draw valid conclusions during this major global event, guidance in collecting data properly is required [4].

This right to reproduction has been discussed thoroughly in the last few years [22]. A right is something to which one is entitled unless trumped by a stronger opposing claim of right. A legal right is arbitrary but enforceable. A moral or ethical right is established by force of argument but is not enforceable unless enacted into law. The debate about why reproductive autonomy is important enough to prioritize along with other morbidities during COVID-19 may be up for discussion. Reproductive autonomy right was and will be a conundrum to all parties involved long before the onset of this pandemic. Nonetheless, it should be noted that autonomy is not privileged in pandemic ethics, while justice is. Once more, it appears that conflict resolution between non-maleficence and respect for autonomy is far more complex and multifaceted.

\section{Bioethical dilemmas practitioners face and attitudes to adopt}

What is certain is that opting to enable pursuing pregnancy requiring assisted reproduction treatment during the pandemic raises valid ethical concerns, which should be navigated employing the five bioethical principles of beneficence, nonmaleficence, veracity, distributive justice, and autonomy [23]. We may be faced with a bioethical dilemma here and be required to achieve a fine balance. On one hand, we should certainly not be discriminating against infertile patients and their need for IVF treatment, while on the other hand, we are called to rise to the highly responsible position of providing services in a safe and effective fashion. From worrying on PPE shortages and staff well-being [24] to the clinician's obligation to the intended parents and children, stress can add up. It is certain that future research will highlight the detrimental effect on the IVF practitioners' well-being - from a psychological perspective - when being called to provide healthcare services during the pandemic [25]. When the common denominator is that pregnancy during the pandemic may be a riskwith so much still in a grey zone-finding the balance is challenging as there may not be a right or wrong answer, a fact that surely fuels debate. From the perspective of the ART field practitioners, it is of paramount importance to abide by the principle of non-maleficence as raised by Hippocratesthe father of medicine - presenting the earliest expression of medical ethics. It appears that even a few months in the pandemic both the practitioners and the patients are still facing a conundrum and in the words of Duzinsky and colleagues, "we may find ourselves exhausted from running a sprint that is in fact turning into a marathon as we speak." In reality, the practice may have to be subject to various ethical standards - at the same time. We may be called to shift priorities from respecting patient autonomy by working towards an individual patient's advantage, to being more concerned for the greater good. It becomes evident that at times as such the concept of patient's autonomy may be jeopardized [24].

Confronting this pandemic, world leaders have introduced the "invisible" war rhetoric to communicate the severity of the COVID-19 public health crisis. Is this a play of war we have been experiencing? Undoubtedly, a pandemic could never be compared to war as ethicists have stated; however, both convey many parallel indirect effects. Profound changes should be expected when great crises arise. In the era of readily available information, patients are enabled to informed decisions and that certainly extends to matters pertaining to pursuing a pregnancy in the time of COVID-19 while its exact impact on pregnancy is still not clearly defined. Fertility treatment patients' attitudes have not yet been reported. Faced with this new fertility treatment reality [3], patients are presented with options. They could proceed taking special precautions during treatment abiding by modified protocols, they could proceed to embryo transfer or cryopreservation of the generated embryos, or they could not pursue treatment altogether until the pandemic situation is resolved. At a time as such, our responsibility principally lies to ensuring an appropriate informed consent to treatment is in place, and this entails the basic principles of informed consent are abided by and medical ethics are taken into consideration. Medical ethics refers to the philosophical approach, aiming to consider issues of sensitive nature pertaining to the essence of a medical action and exerts an inevitable and significant impact on treatment [26]. The value of informed consent for IVF treatment to pursue pregnancy during the pandemic should be underlined and 
appropriate weight should be conveyed. It is the appropriate informed consent that ascertains that both autonomy and integrity are protected while patients are subjected to any medical intervention. Further to that, the informed consent ensures the alliance between patient and practitioner with the IVF professional acting in the patients' best interest medically while respecting their fundamental human rights [27-31]. It is our responsibility - which should be reflected in the informed consent - that albeit patients lack the scientific knowledge, they fully comprehend the disclosed information. Answering a simple question, ticking "yes" or "no," does not make for suitable informed consent material, even though once that may have been the case [30]. It is the shared decision-making process that facilitates disclosure adequacy and guarantees evidence-based, informed, and consistent medical decisions [32]. Nonetheless, ambiguity regarding legal, moral, and clinical perspectives characterizes practical matters pertaining to the concept of risk disclosure [33]. While providing IVF services during a pandemic, we must safeguard a patient-centered approach and convey thorough information in a comprehensive fashion prior to obtaining a signed informed consent. This shall ascertain that we are fulfilling our moral duty to these patients [33].

\section{Conclusion}

While still in the pandemic - although the infection curve appears to have peaked and plateaued in some countries [34] two phases have been identified regarding ART services. The first one dictated cessation of treatment courses during this era of pandemic crisis strengthening the principle of social justice, prioritizing societal needs over individual or organizational desires [3]. Phase one is now followed by the so-called restart phase and IVF treatment proceeds as normal albeit with the respective required modifications to ensure optimal practice. For now, the consensus appears to be "proceed with caution" as fertility treatment is restarted. Studies show that fully modified protocols and codes of conduct are developed to provide a safe practice $[3,5,35]$ abided by both practitioners and patients. Recommendations and guidelines from highly esteemed reproductive societies are in place, and so it appears that we are well prepared and may be in the clear-albeit all recommendations may be subject to future reconsiderations and modifications [36]. Nonetheless, we cannot help but ponder whether perhaps restarting while still in the pandemic may have been premature. On the other hand, similarly to restarting all other socioeconomical aspects of life, perhaps resuming a new kind of normal was anticipated to extend to ART practice equally. The final decision on the appropriateness of the timing between the two phases shall be provided by future data. In the meantime, the requirement for a universal protocol in managing this COVID-19 era in ART may soon become a prerequisite. It is this approach that will enable-for instance - cross border reproductive care to resume in the future towards continuing to serve the influx of patients that for various reasons opt for it. The requirement for certification and quality assurance of services is more demanding than ever. Following recommendations, standards shall follow on appropriate "COVID-19-fit" infrastructures along with a thorough and reliable methodology for patient management. Aside from transparency in ART services provided during the pandemic and the modified protocols recruited to serve the purpose of safe treatment, perhaps ART centers should be audited, approved, and certified to ensure safe practice during this time. The identity of this "authority body" may vary from country to country, as it may be the public or private health sector that may undertake this task.

To conclude, the pandemic has forced us to create a new reality in ART, one where distancing and caution are the protagonists, yet we may fail to define its magnitude and predict its duration, subsequently jeopardizing our ability to determine our future and plan accordingly. We are faced with a new challenge to balance between responding to the committed eagerness of infertile couples to achieve a pregnancy and safeguarding the health of the intended parents and children during the time of this pandemic. The value of an all-inclusive informed consent that would ascertain protecting and promoting the patients' autonomy is highlighted here, along with our ethical, moral, and legal duty towards our patients regarding shared decision-making. The sense of responsibility is more heightened than ever dictating that the path forward should be lit strictly by evidence-based medicine and robust data shaping safe and effective practice.

\section{Compliance with ethical standards}

Conflict of interest The authors declare that they have no conflict of interest

\section{References}

1. Rodriguez-Wallberg KA, Wikander I. A global recommendation for restrictive provision of fertility treatments during the COVID19 pandemic. Acta Obstet Gynecol Scand. Wiley-Blackwell; 2020; 99:569.

2. Requena A, Cruz M, Vergara V, Prados N, Galliano D, Pellicer A. A picture of the covid-19 impact on IVIRMA fertility treatment clinics in Spain and Italy. Reprod Biomed Online. Elsevier; 2020;41:1.

3. Anifandis G, Messini CI, Daponte A, Messinis IE. COVID-19 and fertility: a virtual reality. Reprod Biomed Online [Internet]. 2020 [cited 2020 Jun 29]; Available from: https://www.ncbi.nlm.nih. gov/pmc/articles/PMC7206439/

4. Vermeulen N, Ata B, Gianaroli L, Lundin K, Mocanu E, Rautakallio-Hokkanen S, et al. A picture of medically assisted reproduction activities during the COVID-19 pandemic in Europe. Hum Reprod Open [Internet]. 2020 [cited 2020 Sep 2];2020. 
Available from: https://www.ncbi.nlm.nih.gov/pmc/articles/ PMC7430925/

5. Alviggi C, Esteves SC, Orvieto R, Conforti A, Marca AL, Fischer $\mathrm{R}$, et al. COVID-19 and assisted reproductive technology services: repercussions for patients and proposal for individualized clinical management. Reprod Biol Endocrinol RBE [Internet]. BioMed Central; 2020 [cited 2020 Jun 29];18. Available from: https:// www.ncbi.nlm.nih.gov/pmc/articles/PMC7218705/

6. Berger ZD, Evans NG, Phelan AL, Silverman RD. Covid-19: control measures must be equitable and inclusive. BMJ [Internet]. British Medical Journal Publishing Group; 2020 [cited 2020 Jun 29];368. Available from: https://www.bmj.com/content/368/ bmj.m1141

7. Vaiarelli A, Bulletti C, Cimadomo D, Borini A, Alviggi C, Ajossa S, et al. COVID-19 and ART: the view of the Italian Society of Fertility and Sterility and Reproductive Medicine. Reprod Biomed Online. Elsevier; 2020;40:755-9.

8. Vaiarelli A, Bulletti C, Cimadomo D, Borini A, Argento C, Alviggi $\mathrm{C}$, et al. Are there specific patients candidate for oocyte collection during COVID-19 pandemic? [Internet]. Fertil. Steril. Dialog. 2020 [cited 2020 Aug 30]. Available from: https://www.fertstertdialog. com/posts/64914-are-there-specific-patients-candidate-for-oocytecollection-during-covid-19-pandemic

9. Esteves SC, Lombardo F, Garrido N, Alvarez J, Zini A, Colpi GM, et al. SARS-CoV-2 pandemic and repercussions for male infertility patients: a proposal for the individualized provision of andrological services. Andrology [Internet]. [cited 2020 Jun 29];n/a. Available from: https://onlinelibrary.wiley.com/doi/abs/10.1111/andr.12809

10. Romanski PA, Bortoletto P, Rosenwaks Z, Schattman GL. Delay in IVF treatment up to 180 days does not affect pregnancy outcomes in women with diminished ovarian reserve. Hum Reprod [Internet]. [cited 2020 Jun 23]; Available from: https://academic.oup.com/ humrep/advance-article/doi/10.1093/humrep/deaa137/5858161

11. Vindegaard N, Benros ME. COVID-19 pandemic and mental health consequences: Systematic review of the current evidence. Brain Behav Immun [Internet]. 2020 [cited 2020 Aug 30]; Available from: https://www.ncbi.nlm.nih.gov/pmc/articles/ PMC7260522/

12. Pfefferbaum B, North CS. Mental health and the Covid-19 pandemic. N Engl J Med. Massachusetts Medical Society; 2020;383:510 2 .

13. Curigliano G, Cardoso MJ, Poortmans P, Gentilini O, Pravettoni G, Mazzocco K, et al. Recommendations for triage, prioritization and treatment of breast cancer patients during the COVID-19 pandemic. The Breast. 2020;52:8-16.

14. Preti E, Di Mattei V, Perego G, Ferrari F, Mazzetti M, Taranto P, et al. The psychological impact of epidemic and pandemic outbreaks on healthcare workers: rapid review of the evidence. Curr Psychiatry Rep [Internet]. 2020 [cited 2020 Aug 30];22. Available from: https://www.ncbi.nlm.nih.gov/pmc/articles/PMC7350408/

15. Cousineau TM, Domar AD. Psychological impact of infertility. Best Pract Res Clin Obstet Gynaecol. 2007;21:293-308.

16. Beutel M, Kupfer J, Kirchmeyer P, Kehde S, Köhn F-M, Schroeder-Printzen I, et al. Treatment-related stresses and depression in couples undergoing assisted reproductive treatment by IVF or ICSI. Andrologia. 1999;31:27-35.

17. Quant HS, Zapantis A, Nihsen M, Bevilacqua K, Jindal S, Pal L. Reproductive implications of psychological distress for couples undergoing IVF. J Assist Reprod Genet. 2013;30:1451-8.

18. Turocy J, Robles A, Hercz A, D'Alton M, Forman E, Williams Z. The emotional impact of the ASRM guidelines on fertility patients during the COVID-19 pandemic [Internet]. Fertil. Steril. Dialog. 2020 [cited 2020 Aug 30]. Available from: https://www. fertstertdialog.com/posts/65837-the-emotional-impact-of-theasrm-guidelines-on-fertility-patients-during-the-covid-19pandemic
19. Liang H, Acharya G. Novel corona virus disease (COVID-19) in pregnancy: what clinical recommendations to follow? Acta Obstet Gynecol Scand. 2020;99:439-42.

20. Breslin N, Baptiste C, Miller R, Fuchs K, Goffman D, GyamfiBannerman C, et al. Coronavirus disease 2019 in pregnancy: early lessons. Am J Obstet Gynecol MFM. 2020;100111:2.

21. Chen D, Yang H, Cao Y, Cheng W, Duan T, Fan C, et al. Expert consensus for managing pregnant women and neonates born to mothers with suspected or confirmed novel coronavirus (COVID19) infection. Int J Gynaecol Obstet Off Organ Int Fed Gynaecol Obstet. 2020;149:130-6.

22. Chadwick R. Reproductive autonomy and responsibility: current trends. Bioethics. 2018;32:2.

23. Dialog F and S. Core bioethical principles and the ethics of continued care during the COVID-19 war [Internet]. Fertil. Steril. Dialog. 2020 [cited 2020 Jun 29]. Available from: http://www. fertstertdialog.com/users/16110-fertility-and-sterility/posts/66118core-bioethical-principles-and-the-ethics-of-continued-care-duringthe-covid-19-war

24. Dudzinski DM, Hoisington BY, Brown CE. Ethics lessons from Seattle's early experience with COVID-19. Am J Bioeth. Taylor \& Francis; 2020;20:1-8.

25. Elbay RY, Kurtulmuș A, Arpacioğlu S, Karadere E. Depression, anxiety, stress levels of physicians and associated factors in Covid19 pandemics. Psychiatry Res. 2020;290:113130.

26. Markose A, Krishnan R, Ramesh M. Medical ethics. J Pharm Bio Allied Sci. 2016;8:S1-4.

27. Percival T. Medical ethics: or, a code of institutes and precepts, adapted to the professional conduct of physicians and surgeons : to which is added an appendix ; containing a discourse on hospital duties ; also notes and illustrations. S. Russell; 1803.

28. Bartholome WG. A history and theory of informed consent. In: Faden RR, Beauchamp TL, editors. Ethics, vol. 98. Chicago: The University of Chicago Press; 1988. p. 605-6.

29. Murray PM. The history of informed consent. Iowa Orthop J. 1990;10:104-9.

30. Jones JW, McCullough LB, Richman BW. Informed consent: it's not just signing a form. Thorac Surg Clin. Elsevier; 2005;15:45160 .

31. Chan SW, Tulloch E, Cooper ES, Smith A, Wojcik W, Norman JE. Montgomery and informed consent: where are we now? BMJ [Internet]. British Medical Journal Publishing Group; 2017 [cited 2020 May 23];357. Available from: https://www.bmj.com/content/ 357/bmj.j2224

32. Katz SJ, Hawley $\mathrm{S}$. The value of sharing treatment decision making with patients: expecting too much? JAMA. 2013;310:1559-60.

33. Leonard C, Toner J. The physician's duty to warn their patients about the risks associated with medical intervention: a review and discussion. Ulster Med J. 2019;88:1-3.

34. Block P, Hoffman M, Raabe IJ, Dowd JB, Rahal C, Kashyap R, et al. Social network-based distancing strategies to flatten the COVID-19 curve in a post-lockdown world. Nat Hum Behav. 2020;4:588-96.

35. Arav A. A recommendation for IVF lab practice in light of the current COVID-19 pandemic. J Assist Reprod Genet. 2020;1.

36. De Santis L, Anastasi A, Cimadomo D, Klinger FG, Licata E, Pisaturo V, et al. COVID-19: the perspective of Italian embryologists managing the IVF laboratory in pandemic emergency. Hum Reprod Oxf Engl [Internet]. 2020 [cited 2020 Jun 29]; Available from: https://www.ncbi.nlm.nih.gov/pmc/articles/PMC7184410/

Publisher's note Springer Nature remains neutral with regard to jurisdictional claims in published maps and institutional affiliations. 